\title{
GRAPHS DETERMINED BY THEIR (SIGNLESS) LAPLACIAN SPECTRA*
}

\author{
MUHUO LIU ${ }^{\dagger}$, BOLIAN LIU ${ }^{\ddagger}$, AND FUYI WEI ${ }^{\dagger}$
}

\begin{abstract}
Let $S(n, c)=K_{1} \vee\left(c K_{2} \cup(n-2 c-1) K_{1}\right)$, where $n \geq 2 c+1$ and $c \geq 0$. In this paper, $S(n, c)$ and its complement are shown to be determined by their Laplacian spectra, respectively. Moreover, we also prove that $S(n, c)$ and its complement are determined by their signless Laplacian spectra, respectively.
\end{abstract}

Key words. Laplacian spectrum, Signless Laplacian spectrum, Complement graph.

AMS subject classifications. 05C50, 15A18, 15A36.

1. Introduction. In this paper, $G=(V, E)$ is an undirected simple graph. The neighbor set of a vertex $u$ is denoted by $N(u)$. Let $d(u)$ be the degree of vertex $u$, namely, $d(u)=|N(u)|$. If $d(u)=1$, then $u$ is called a pendant vertex of $G$. Suppose the degree of vertex $v_{i}$ equals $d_{i}$, for $i=1,2, \ldots, n$. Throughout this paper, we enumerate the degrees in non-increasing order, i.e., $d_{1} \geq d_{2} \geq \cdots \geq d_{n}$. Sometimes we write $d_{i}(G)$ in place of $d_{i}$, in order to indicate the dependence on $G$. By $v_{1} v_{2} \in E(G)$, we mean an edge, of which the end vertices are $v_{1}$ and $v_{2}$. Let $G_{1} \cup G_{2}$ denote the (disconnected) graph consisting of two components $G_{1}$ and $G_{2}$, and $k G$ be the graph consisting of $k$ (where $k \geq 0$ is an integer) copies of the graph $G$. The join $G_{1} \vee G_{2}$ of two disjoint graphs $G_{1}$ and $G_{2}$ is the graph having vertex set $V\left(G_{1} \vee G_{2}\right)=V\left(G_{1} \cup G_{2}\right)$ and edge set $E\left(G_{1} \vee G_{2}\right)=E\left(G_{1}\right) \cup E\left(G_{2}\right) \cup\left\{u v: u \in V\left(G_{1}\right), v \in V\left(G_{2}\right)\right\}$. As usual, $K_{n}, P_{n}$ and $C_{n}$ denote the complete graph, path and cycle of order $n$, respectively. Specially, $K_{1}$ denotes an isolated vertex. A graph is a cactus, or a treelike graph, if any pair of its cycles has at most one common vertex $[1,20]$. If all cycles of the cactus $G$ have exactly one common vertex, then $G$ is called a bundle [1]. Let $S(n, c)$ be the bundle with $n$ vertices and $c$ cycles of length 3 depicted in Figure 1.1, where $n \geq 2 c+1$ and $c \geq 0$. By the definition, it follows that $S(n, c)=K_{1} \vee\left(c K_{2} \cup(n-2 c-1) K_{1}\right)$.

*Received by the editors on February 10, 2010. Accepted for publication on January 31, 2011. Handling Editor: Bryan Shader. This work is supported by the Foundation for Distinguished Young Talents in Higher Education of Guangdong, China (No. LYM10039) and NNSF of China (No. 11071088).

${ }^{\dagger}$ Department of Applied Mathematics, South China Agricultural University, Guangzhou, 510642, P.R. China.

${ }^{\ddagger}$ School of Mathematic Science, South China Normal University, Guangzhou, 510631, P.R. China (liubl@scnu.edu.cn; Bolian Liu). 


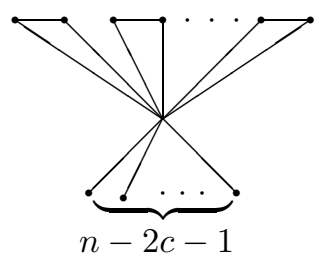

FIG. 1.1. The bundle $S(n, c)$.

The adjacency matrix $A(G)=\left[a_{i j}\right]$ of $G$ is an $n \times n$ symmetric matrix of 0's and 1 's with $a_{i j}=1$ if and only if $v_{i} v_{j} \in E(G)$. Let $D(G)$ be the diagonal matrix whose $(i, i)$-entry is $d_{i}$, where $1 \leq i \leq n$. The Laplacian matrix of $G$ is $L(G)=D(G)-A(G)$, and the signless Laplacian matrix of $G$ is $Q(G)=D(G)+A(G)$. Sometimes, $Q(G)$ is also called the unoriented Laplacian matrix of $G$ (see, e.g., $[10,22]$ ).

It is well known that $L(G)$ is positive semidefinite so that its eigenvalues can be arranged as follows:

$$
\lambda_{1}(G) \geq \lambda_{2}(G) \geq \cdots \geq \lambda_{n}(G)=0
$$

Research on the signless Laplacian matrix has recently become popular $[3,5$, $10,22]$. It is easy to see that $Q(G)$ is also positive semidefinite [5] and hence its eigenvalues can be arranged as:

$$
\mu_{1}(G) \geq \mu_{2}(G) \geq \cdots \geq \mu_{n}(G) \geq 0
$$

If there is no confusion, sometimes we write $\lambda_{i}(G)$ as $\lambda_{i}$, and $\mu_{i}(G)$ as $\mu_{i}$. In the following, let $S L(G)$ and $S Q(G)$ denote the spectra, i.e., eigenvalues of $L(G)$ and $Q(G)$, respectively.

A graph $G$ is said to be determined by its Laplacian spectrum (resp. adjacency spectrum, signless Laplacian spectrum) if there does not exist a non-isomorphic graph $H$ such that $H$ and $G$ share the same Laplacian spectrum (resp. adjacency spectrum, signless Laplacian spectrum). The question "which graphs are determined by their spectra?" is proposed by van Dam and Haemers in [6]. Up to now, only a few families of graphs are known to be determined by their spectra [6,9]. For example, the path, the complement of a path, the complete graph, and the cycle were proved to be determined by their adjacency spectra $[6,9]$. The path, the complete graph, the cycle, the star and some quasi-star graphs, together with their complement graphs were shown to be determined by their Laplacian spectra $[6,9,15,21]$. Let $K_{n}^{m}$ be the graph obtained by attaching $m$ pendant vertices to a vertex of the complete graph $K_{n-m}$, and $U_{n, p}$ be the graph obtained by attaching $n-p$ pendant vertices to a vertex of $C_{p}$. Recently, Zhang and Zhang in [23] confirmed that $K_{n}^{m}$ together with its 
complement are determined by their Laplacian and adjacency spectra, respectively, and $U_{n, p}$ is determined by its Laplacian spectrum. Moreover, they proved that $U_{n, p}$ is determined by its adjacency spectrum if $p$ is odd. Very recently, the authors of [24] showed that $H_{n, p}$, which is obtained by appending a cycle $C_{p}$ to a pendant vertex of a path $P_{n-p}$, is determined by its signless Laplacian spectrum.

$S(n, c)$ is an extremal graph in some classes of graphs. For instance, $S(n, c)$ is the graph with the maximal spectral radius, the maximal Merrifield-Simmons index, the minimal Hosoya index, the minimal Wiener index, and the minimal Randic index in the set of all connected cacti on $n$ vertices with $c$ cycles $[1,14]$. In this paper, by using a new method different from $[6,9,15,21,23,24]$, we show that $S(n, c)$ together with its complement are determined by their Laplacian spectra, and we also prove that $S(n, c)$ together with its complement are determined by their signless Laplacian spectra.

2. $S(n, c)$ and its complement are determined by their Laplacian spectra. The following lemmas are well-known:

LEMma 2.1. [12, 18] If $G_{1}$ and $G_{2}$ are two disjoint graphs on $k$ and $m$ vertices respectively, with Laplacian eigenvalues $0=\lambda_{k}\left(G_{1}\right) \leq \lambda_{k-1}\left(G_{1}\right) \leq \cdots \leq \lambda_{1}\left(G_{1}\right)$ and $0=\lambda_{m}\left(G_{2}\right) \leq \lambda_{m-1}\left(G_{2}\right) \leq \cdots \leq \lambda_{1}\left(G_{2}\right)$ respectively, then the Laplacian eigenvalues of $G_{1} \vee G_{2}$ are given by $0, \lambda_{k-1}\left(G_{1}\right)+m, \ldots, \lambda_{1}\left(G_{1}\right)+m, \lambda_{m-1}\left(G_{2}\right)+k, \ldots, \lambda_{1}\left(G_{2}\right)+k$, and $m+k$.

Lemma 2.2. [13] If $G=(V, E)$ is a graph of order $n$, then $\lambda_{1}(G) \leq n$. Moreover, $\lambda_{1}(G)=n \geq 2$ if and only if $G=G_{1} \vee G_{2}$, where each of $G_{1}$ and $G_{2}$ has at least one vertex.

Let $G^{\prime}=G+e$ be the graph obtained from $G$ by inserting a new edge $e$ into $G$, and $G-u$ be the graph obtained from $G$ by deleting the vertex $u \in V(G)$ and all the edges adjacent to $u$. It follows by the Courant-Weyl inequalities [4, Theorem 2.1] that:

Lemma 2.3. [7] The Laplacian eigenvalues of $G$ and $G^{\prime}=G+e$ interlace, that is, $\lambda_{1}\left(G^{\prime}\right) \geq \lambda_{1}(G) \geq \lambda_{2}\left(G^{\prime}\right) \geq \lambda_{2}(G) \geq \cdots \geq \lambda_{n}\left(G^{\prime}\right)=\lambda_{n}(G)=0$.

LEMMA 2.4. [17, 19] If $G$ is a graph with $n$ vertices and at least one edge, then $\mu_{1}(G) \geq \lambda_{1}(G) \geq d_{1}(G)+1$. If $G$ is connected, the first equality holds if and only if $G$ is bipartite, the second equality holds if and only if $d_{1}(G)=n-1$.

As usual, $K_{s, t}$ denotes the complete bipartite graph with $s$ vertices in one part and $t$ in the other. Specially, $K_{1, n-1}$ denotes the star of order $n$. By Lemmas 2.1-2.2, it is not difficult to prove that: 
Lemma 2.5. [15, 21] $K_{1, n-1}$ is determined by its Laplacian spectrum.

THEOREM 2.6. If $c \geq 0$, then $S(n, c)$ is determined by its Laplacian spectrum.

Proof. If $c=0$, then $S(n, c) \cong K_{1, n-1}$. By Lemma 2.5, the result follows. In the following, assume that $c \geq 1$. Since $n \geq 2 c+1 \geq c+2, n=c+2$ if and only if $n=3$ and $c=1$. Thus, $n=c+2$ implies that $S(n, c) \cong C_{3}$, it can be readily checked that $C_{3}$ is determined by its Laplacian spectrum [6]. So, we may assume that $c \geq 1$ and $n>c+2$ in the sequel.

By Lemma 2.1 and $S L\left(K_{2}\right)=(2,0)$, we have

$$
S L(S(n, c))=(n, 3, \ldots, 3,1, \ldots, 1,0),
$$

where the multiplicity of 3 is $c$, and the multiplicity of 1 is $n-c-2$. Now suppose there exists some graph $G$, such that $S L(G)=S L(S(n, c))$, then $\lambda_{1}(G)=n$. By Lemma 2.2, it follows that $G=G_{1} \vee G_{2}$, where $G_{1}$ and $G_{2}$ are two disjoint graphs with $\left|V\left(G_{1}\right)\right| \geq\left|V\left(G_{2}\right)\right|$. Since $n>c+2$, we have $\lambda_{n-1}(G)=\lambda_{n-1}(S(n, c))=1$.

Next we shall prove that $\left|V\left(G_{2}\right)\right|=1$. Otherwise, if $\left|V\left(G_{2}\right)\right| \geq 2$, by Lemmas 2.1 and 2.3, we can conclude that $\lambda_{n-1}(G) \geq \lambda_{n-1}\left(K_{\left|V\left(G_{1}\right)\right|,\left|V\left(G_{2}\right)\right|}\right)=\left|V\left(G_{2}\right)\right| \geq 2$, a contradiction. Thus, $\left|V\left(G_{2}\right)\right|=1$ follows. Now suppose $V\left(G_{2}\right)=\left\{v_{0}\right\}$, then $G_{1}=G-$ $v_{0}$. By Lemma 2.1 and $S L(G)=S L(S(n, c))$, then $S L\left(G_{1}\right)=(2,2, \ldots, 2,0,0, \ldots, 0)$, where the multiplicity of 2 is $c$, and the multiplicity of 0 is $n-c-1$. By Lemma 2.4, we can conclude that $d_{1}\left(G_{1}\right)=1$, and hence $G_{1}=c K_{2} \cup(n-2 c-1) K_{1}$. Therefore, $G \cong S(n, c)$.

Let $G^{C}$ be the complement graph of $G$. In particular, $S^{C}(n, c)$ denotes the complement graph of $S(n, c)$. For the relation between $S L(G)$ and $S L\left(G^{C}\right)$, it has been shown that:

LEMMA 2.7. [17] Let $G$ be a graph with $n$ vertices. If $\lambda_{i}(G), i=1,2, \ldots, n$ are the eigenvalues of $L(G)$, then the eigenvalues of $L\left(G^{C}\right)$ are $n-\lambda_{i}(G), i=1,2, \ldots, n-1$ and 0 .

By Lemma 2.7 and Theorem 2.6, we have:

Corollary 2.8. If $c \geq 0$, then $S^{C}(n, c)$ is determined by its Laplacian spectrum.

3. $S(n, c)$ is determined by its signless Laplacian spectrum. In this section, we shall show that $S(n, c)$ is determined by its signless Laplacian spectrum. First we need some lemmas.

Suppose $M$ and $N$ are real symmetric matrices of order $n$ and $m$ with eigenvalues $\rho_{1}(M) \geq \cdots \geq \rho_{m}(M)$ and $\rho_{1}(N) \geq \cdots \geq \rho_{n}(N)$, respectively. It is well-known that: 
LEMma 3.1. [11] If $M$ is a principal submatrix of $N$, then the eigenvalues of $M$ interlace those of $N$, i.e., $\rho_{i}(N) \geq \rho_{i}(M) \geq \rho_{n-m+i}(N)$ for $i=1,2, \ldots, m$.

LEMMA 3.2. [8] If $G$ is a graph on $n$ vertices with vertex degrees $d_{1} \geq d_{2} \geq$ $\cdots \geq d_{n}$ and signless Laplacian eigenvalues $\mu_{1} \geq \mu_{2} \geq \cdots \geq \mu_{n}$, then $\mu_{2} \geq d_{2}-1$. Moreover, if $\mu_{2}=d_{2}-1$, then $d_{1}=d_{2}$, and the maximum and the second maximum degree vertices are adjacent.

By Lemmas 2.4 and 3.2 , it follows that $\mu_{1} \geq d_{1}+1$ and $\mu_{2} \geq d_{2}-1$. For the general case, we have:

THEOREM 3.3. If $G$ is a finite simple graph on $n$ vertices with vertex degrees $d_{1} \geq d_{2} \geq \cdots \geq d_{n}$ and signless Laplacian eigenvalues $\mu_{1} \geq \mu_{2} \geq \cdots \geq \mu_{n}$, then $\mu_{m} \geq d_{m}-m+1$, where $m=1,2, \ldots, n$.

To prove Theorem 3.3, we need the next lemma.

Lemma 3.4. [4] (Weyl) Suppose $A_{n}$ and $B_{n}$ are two real symmetric matrices of order $n$, then $\rho_{n}(A)+\rho_{n}(B) \leq \rho_{n}(A+B)$, where $\rho_{n}(A), \rho_{n}(B)$ and $\rho_{n}(A+B)$ denote the smallest eigenvalues of $A, B$ and $A+B$, respectively.

Proof of Theorem 3.3. Since $Q(G)$ is positive semidefinite, $\mu_{m} \geq 0$. If $d_{m}-m+1 \leq$ 0 , the result already holds. So, we assume that $d_{m}>m-1$ in the following.

Let $T=\left\{v_{1}, v_{2}, \ldots, v_{m}\right\}$. Consider the principal submatrix $Q_{T}$ of $Q(G)$ with rows and columns indexed by $T$. Let $Q(T)$ be the signless Laplacian matrix of the subgraph induced by $T$. Then, $Q_{T}=Q(T)+D^{\prime}(T)$, where $D^{\prime}(T)$ is the diagonal matrix and the $(i, i)$-entry of $D^{\prime}(T)$ is the number of neighbors of $v_{i}$ outside $T$. Since $Q(T)$ is positive semidefine, and $D^{\prime}(T) \geq\left(d_{m}-m+1\right) I_{m}$, by Lemma 3.4 we have $\rho_{m}\left(Q_{T}\right) \geq \rho_{m}(Q(T))+\rho_{m}\left(D^{\prime}(T)\right) \geq \rho_{m}\left(D^{\prime}(T)\right) \geq d_{m}-m+1$. Recall that $Q_{T}$ is the principal submatrix of $Q(G)$, thus Lemma 3.1 implies that $\mu_{m} \geq \rho_{m}\left(Q_{T}\right) \geq$ $d_{m}-m+1$. We get the required inequality.

REMARK 3.5. The main idea of the proof in Theorem 3.3 comes from Lemma 2 of [2]. In [2], it has been shown that "Let $G$ be a finite simple graph on $n$ vertices with vertex degree $d_{1} \geq d_{2} \geq \cdots \geq d_{n}$ and Laplacian eigenvalues $\lambda_{1} \geq \lambda_{2} \geq \cdots \geq \lambda_{n}$. If $G \not K_{m} \cup(n-m) K_{1}$, then $\lambda_{m} \geq d_{m}-m+2$, where $m=1,2, \ldots, n$." Though $\mu_{1} \geq \lambda_{1} \geq d_{1}+1$ by Lemma $2.4, \mu_{m} \geq d_{m}-m+2$ does not hold for all connected graphs. For example, $\mu_{2}\left(K_{n}-e\right)=n-2<n-1=d_{2}\left(K_{n}-e\right)$, where $K_{n}-e$ is the graph obtained from $K_{n}$ by deleting one edge and $n \geq 4$.

Let $\Phi(G, x)=\operatorname{det}(x I-Q(G))$ be the signless Laplacian characteristic polynomial of $G$.

LEMMA 3.6. If $c \geq 1$, then $\mu_{1}(S(n, c))>n, \mu_{2}(S(n, c)) \leq 3$ and $0<\mu_{n}(S(n, c))$ 
$\leq 1$

Proof. By a straightforward computation, we have

$$
\Phi(S(n, c), x)=(x-1)^{n-c-2}(x-3)^{c-1} \varphi_{1}(x),
$$

where $\varphi_{1}(x)=x^{3}-(n+3) x^{2}+3 n x-4 c$.

We consider the next two cases.

Case $1 . n \geq 2 c+2$.

Since $\varphi_{1}(0)=-4 c<0, \varphi_{1}(1)=2(n-2 c-1)>0, \varphi_{1}(3)=-4 c<0, \varphi_{1}(n)=$ $-4 c<0$ and $\varphi_{1}(n+1)=n^{2}-n-2-4 c \geq n^{2}-n-2-2 n+4=n^{2}-3 n+2>0$. By Eq. (3.1), it follows that $\mu_{1}(S(n, c))>n, \mu_{2}(S(n, c)) \leq 3$ and $0<\mu_{n}(S(n, c))<1$.

Case 2. $n=2 c+1$.

If $c=1$, then $n=3$ and hence $S(n, c)=C_{3}$, it is easily checked the result follows. Thus, we may suppose that $n \geq 5$, i.e., $c \geq 2$ in the following. Then, Eq. (3.1) can be rewritten as

$$
\Phi(S(n, c), x)=(x-1)^{n-c-1}(x-3)^{c-1} \varphi_{2}(x),
$$

where $\varphi_{2}(x)=x^{2}-(n+2) x+4 c$.

Since $\varphi_{2}(1)=2 c-2>0, \varphi_{2}(2)=-2<0, \varphi_{2}(n)=-2<0$ and $\varphi_{2}(n+1)=$ $2 c-2>0$. By Eq. (3.2), it follows that $\mu_{1}(S(n, c))>n, \mu_{2}(S(n, c))=3$ and $\mu_{n}(S(n, c))=1$.

By combining the above arguments, the result follows.

Lemma 3.7. [5] Let $G=(V, E)$ be a graph on $n$ vertices. Then, $\mu_{1}(G) \leq$ $\max \{d(u)+d(v): u v \in E\}$. For a connected graph $G$, equality holds if and only if $G$ is regular or semi-regular bipartite.

LEMMA 3.8. For $c \geq 1$, if $S Q(G)=S Q(S(n, c))$, then $G$ is connected with $d_{2}(G) \leq 4$. Moreover, $d_{2}(G)=4$ implies that $d_{1}(G)=d_{2}(G)$.

Proof. Since $S Q(G)=S Q(S(n, c))$, by Lemma 3.6 it follows that $\mu_{1}(G)=$ $\mu_{1}(S(n, c))>n$ and $\mu_{2}(G)=\mu_{2}(S(n, c)) \leq 3$. By Lemma 3.2, we can conclude that $d_{2}(G) \leq 4$, and $d_{2}(G)=4$ implies that $d_{1}(G)=d_{2}(G)$.

Suppose to the contrary that $G$ is disconnected. Let $G_{1}$ be the greatest connected component, i.e., the connected component with largest number of vertices, of $G$. Since $d_{2}(G) \leq 4$ and $\mu_{1}(G)>n$, we have $n-3 \leq d_{1}(G) \leq n-2$ by Lemma 3.7. We consider the next two cases.

Case 1. $d_{1}(G)=n-3$. 
Then, $\left|V\left(G_{1}\right)\right| \geq n-2$. If $\left|V\left(G_{1}\right)\right|=n-1$, then $G=G_{1} \cup K_{1}$. This implies that $\mu_{n}(G)=0$, a contradiction to $\mu_{n}(G)=\mu_{n}(S(n, c))>0$. If $\left|V\left(G_{1}\right)\right|=n-2$, then $G=G_{1} \cup K_{2}$ or $G=G_{1} \cup 2 K_{1}$. This also implies that $\mu_{n}(G)=0$, a contradiction.

Case 2. $d_{1}(G)=n-2$.

Then, $\left|V\left(G_{1}\right)\right|=n-1$, and hence $G=G_{1} \cup K_{1}$. This also implies that $\mu_{n}(G)=0$, a contradiction to $\mu_{n}(G)=\mu_{n}(S(n, c))>0$.

Thus, $G$ is connected.

Let $m(v)$ denote the average of the degree of the vertices adjacent to $v$, i.e., $m(v)=\sum_{u \in N(v)} d(u) / d(v)$.

Lemma 3.9. [7] Let $G$ be a connected graph. Then $\mu_{1}(G) \leq \max \{d(v)+m(v)$ : $v \in V\}$, and equality holds if and only if $G$ is a regular graph or a semi-regular bipartite graph.

LEMmA 3.10. Let $G=(V, E)$ be a connected graph on $n \geq 2 c+3$ vertices with $n+c-1$ edges. If $c \geq 1$ and $d_{1}(G) \leq n-2$, then $\mu_{1}(G) \leq n$.

Proof. By Lemma 3.9, we only need to prove that $\max \{d(v)+m(v): v \in V\} \leq n$. Suppose $\max \{d(v)+m(v): v \in V\}$ occurs at the vertex $u_{0}$. Three cases arise: $d\left(u_{0}\right)=1, d\left(u_{0}\right)=2$, or $3 \leq d\left(u_{0}\right) \leq n-2$.

Case 1. $d\left(u_{0}\right)=1$.

Suppose $v \in N\left(u_{0}\right)$. Since $d(v) \leq d_{1}(G) \leq n-2, d\left(u_{0}\right)+m\left(u_{0}\right)=d\left(u_{0}\right)+d(v) \leq$ $n-1<n$.

Case 2. $d\left(u_{0}\right)=2$.

Suppose that $v, w \in N\left(u_{0}\right)$.

If $v w \in E$, since $G$ is a connected graph with $n+c-1$ edges, it follows that $|N(v) \cap N(w)| \leq c$ and $|N(v) \cup N(w)| \leq n$. Therefore, $d\left(u_{0}\right)+m\left(u_{0}\right)=2+\frac{d(v)+d(w)}{2} \leq$ $2+\frac{n+c}{2} \leq n$ by $n \geq 2 c+3$.

If $v w \notin E$, since $G$ is a connected graph with $n+c-1$ edges, it follows that $|N(v) \cap N(w)| \leq c+1$ and $|N(v) \cup N(w)| \leq n-2$. Therefore, $d\left(u_{0}\right)+m\left(u_{0}\right)=$ $2+\frac{d(v)+d(w)}{2} \leq 2+\frac{n+c-1}{2}<n$ by $n \geq 2 c+3$.

Case $3.3 \leq d\left(u_{0}\right) \leq n-2$.

Note that $3 \leq d\left(u_{0}\right) \leq n-2$ and the number of edges of $G$ is $n+c-1$, then $d\left(u_{0}\right)+m\left(u_{0}\right) \leq \bar{d}\left(u_{0}\right)+\frac{2(n+c-1)-d\left(u_{0}\right)-1}{d\left(u_{0}\right)}=d\left(u_{0}\right)-1+\frac{2 n+2 c-3}{d\left(u_{0}\right)}$. Next we shall prove that $d\left(u_{0}\right)-1+\frac{2 n+2 c-3}{d\left(u_{0}\right)} \leq n$, equivalently, $d\left(u_{0}\right)\left(n+1-d\left(u_{0}\right)\right) \geq 2 n+2 c-3$. Let 
$f(x)=(n+1-x) x$.

When $3 \leq x \leq \frac{n+1}{2}$, since $f^{\prime}(x)=n+1-2 x \geq 0$, we have $f(x) \geq f(3)=$ $3(n-2) \geq 2 n+2 c-3$ by $n \geq 2 c+3$.

When $\frac{n+1}{2} \leq x \leq n-2$, since $f^{\prime}(x)=n+1-2 x \leq 0$, we have $f(x) \geq f(n-2)=$ $3(n-2) \geq 2 n+2 c-3$ by $n \geq 2 c+3$.

By combining the above arguments, the conclusion follows.

LEMma 3.11. [5] Let $G$ be a graph with $n$ vertices, $m$ edges. We have $\sum_{i=1}^{n} \mu_{i}=$ $\sum_{i=1}^{n} d_{i}=2 m$, and $\sum_{i=1}^{n} \mu_{i}^{2}=2 m+\sum_{i=1}^{n} d_{i}^{2}$.

Lemma 3.12. For $c \geq 1$, if $n=2 c+2$ or $n=2 c+1$, then there does not exist any connected graph $G$ on $n$ vertices with $n+c-1$ edges and $d_{1}(G) \leq n-2$ such that $S Q(G)=S Q(S(n, c))$.

Proof. Here we only prove the case of $n=2 c+2$, because the proof of $n=2 c+1$ is analogous. When $3 \leq n \leq 7$, it is easily checked the result follows by the aid of computer. Thus, we may assume that $n \geq 8$ in the following. Suppose to the contrary, there exists some connected graph $G$ on $n=2 c+2$ vertices with $n+c-1$ edges and $d_{1}(G) \leq n-2$ such that $S Q(G)=S Q(S(n, c))$. By Lemmas 3.6-3.8, we can conclude that $d_{2}(G) \leq 4$ and $n-3 \leq d_{1}(G) \leq n-2$ because $\mu_{1}(G)=\mu_{1}(S(n, c))>n$. We divide the proof into the next two cases.

Case 1. $d_{1}(G)=n-3$.

If $d_{2}(G) \leq 3$, then Lemma 3.7 implies that $\mu_{1}(G) \leq n<\mu_{1}(S(n, c))$, a contradiction. Thus, $d_{2}(G)=4$. So Lemma 3.8 implies that $d_{1}(G)=d_{2}(G)$, and hence $n=7$, a contradiction to the fact that $n \geq 8$.

Case 2. $d_{1}(G)=n-2$.

If $d_{2}(G) \leq 2$, then Lemma 3.7 implies that $\mu_{1}(G) \leq n<\mu_{1}(S(n, c))$, a contradiction. If $d_{2}(G)=4$, Lemma 3.8 implies that $d_{1}(G)=d_{2}(G)$, and hence $n=6$, a contradiction. Thus, $d_{2}(G)=3$. Suppose $G$ has $x$ vertices of degree $3, y$ vertices of degree 2. Then, $G$ has $n-x-y-1$ pendant vertices. By Lemma 3.11, it follows that

$$
\left\{\begin{array}{l}
n-2+3 x+2 y+n-x-y-1=2 n+2 c-2 \\
(n-2)^{2}+9 x+4 y+n-x-y-1=(n-1)^{2}+8 c+n-2 c-1 .
\end{array}\right.
$$

By Eqs. (3.3) and $n=2 c+2$, we have $x=n-3$ and $y=5-n<0$, a contradiction.

By combining the above arguments, this completes the proof of this result.

LEMMA 3.13. [5] In any graph, the multiplicity of the eigenvalue 0 of the signless Laplacian is equal to the number of bipartite components. Moreover, the least eigen- 
value of the signless Laplacian of a connected graph is equal to 0 if and only if the graph is bipartite. In this case, 0 is a simple eigenvalue.

LEMMA 3.14. If $n \neq 4$, then $K_{1, n-1}$ is determined by its signless Laplacian spectrum.

Proof. Suppose there exists some graph $G$ such that $S Q(G)=S Q\left(K_{1, n-1}\right)$. It is well-known that if $G$ is bipartite graph, then $S Q(G)=S L(G)$ (see [5]). Thus, $S Q\left(K_{1, n-1}\right)=S L\left(K_{1, n-1}\right)=(n, 1,1, \ldots, 1,0)$, where the multiplicity of 1 is $n-2$. By Lemma 3.2, we have $d_{2}(G)-1 \leq \mu_{2}(G)=\mu_{2}\left(K_{1, n-1}\right)=1$. So, $d_{2}(G) \leq 2$.

If $G$ is connected, since $\mu_{n}(G)=\mu_{n}\left(K_{1, n-1}\right)=0$, by Lemma 3.13, $G$ is connected bipartite, and hence $S L(G)=S Q(G)=S L\left(K_{1, n-1}\right)$. By Lemma 2.5, it follows that $G \cong K_{1, n-1}$.

If $G$ is disconnected, by Lemma 3.7, we have $d_{1}(G)=n-2$ and $d_{2}(G)=2$ by $\mu_{1}(G)=n$. Moreover, Lemma 3.2 implies that $n-2=d_{1}(G)=d_{2}(G)=2$, and hence $n=4$, a contradiction.

REMARK 3.15. It is easily checked that $S Q\left(K_{1,3}\right)=S Q\left(K_{3} \cup K_{1}\right)$. Thus, $S(n, c)$ is not determined by its signless Laplacian spectrum when $c=0$ and $n=4$.

TheOREm 3.16. Suppose $c \geq 0$, then $S(n, c)$ is determined by its signless Laplacian spectrum except for the case of $c=0$ and $n=4$.

Proof. If $c=0$, then $S(n, c) \cong K_{1, n-1}$. By Lemma 3.14 and Remark 3.15, the result follows. Next we assume that $c \geq 1$. Now suppose there exists some graph $G$ such that $S Q(G)=S Q(S(n, c))$. Lemmas 3.8 and 3.11 imply that $G$ is connected and $\sum_{i=1}^{n} d_{i}(G)=2(n+c-1)$. Thus, $G$ has $n+c-1$ edges. By Lemmas 3.8, 3.10 and 3.12 , we can conclude that $G$ is a connected graph with $d_{1}(G)=n-1$ and $d_{2}(G) \leq 4$ because $\mu_{1}(G)=\mu_{1}(S(n, c))>n$. Suppose $G$ has $x$ vertices of degree $4, y$ vertices of degree $3, z$ vertices of degree 2 . Then, $G$ has $n-x-y-z-1$ pendant vertices. By Lemma 3.11, it follows that

(3.4) $\left\{\begin{array}{l}n-1+4 x+3 y+2 z+n-x-y-z-1=2 n+2 c-2 \\ (n-1)^{2}+16 x+9 y+4 z+n-x-y-z-1=(n-1)^{2}+8 c+n-2 c-1 .\end{array}\right.$

By Eqs. (3.4), we have $6 x+2 y=0$. Thus, $x=y=0$ and $z=2 c$. Note that $d_{1}(G)=n-1$. Then, $G \cong S(n, c)$ follows.

4. $S^{C}(n, c)$ is determined by its signless Laplacian spectrum. In this section, we shall show that $S^{C}(n, c)$ is determined by its signless Laplacian spectrum. We list more lemmas as follows.

LEMma 4.1. [3] The signless Laplacian eigenvalues of $G$ and $G^{\prime}=G+e$ interlace, that is, $\mu_{1}\left(G^{\prime}\right) \geq \mu_{1}(G) \geq \mu_{2}\left(G^{\prime}\right) \geq \mu_{2}(G) \geq \cdots \geq \mu_{n}\left(G^{\prime}\right) \geq \mu_{n}(G) \geq 0$. 
Lemma 4.2. [16] Suppose $G$ has $n$ vertices and $d_{n}$ is the minimum degree of vertices of $G$, then $\mu_{n} \leq d_{n}$.

Lemma 4.3. If $c \geq 1$ and $n \geq 7$, then $\mu_{n}\left(S^{C}(n, c)\right)=0, \mu_{n-1}\left(S^{C}(n, c)\right) \geq n-5$, $\mu_{2}\left(S^{C}(n, c)\right)=n-3$ and $\mu_{1}\left(S^{C}(n, c)\right) \geq 2(n-3)$.

Proof. By a straightforward computation, we have

$$
\Phi\left(S^{C}(n, c), x\right)=x(x-n+5)^{c-1}(x-n+3)^{n-c-2} \varphi_{3}(x),
$$

where $\varphi_{3}(x)=x^{2}-3(n-3) x+2\left(n^{2}-7 n+10+2 c\right)$.

It is easy to see that the roots of $\varphi_{3}(x)=0$ are

$$
\frac{3(n-3) \pm \sqrt{(n+1)^{2}-16 c}}{2} .
$$

Note that $n \geq 2 c+1$. Then,

$$
\begin{gathered}
\mu_{1}=\frac{3(n-3)+\sqrt{(n+1)^{2}-16 c}}{2} \geq 2(n-3), \\
\text { and } \quad n-5<\frac{3(n-3)-\sqrt{(n+1)^{2}-16 c}}{2} \leq n-3 .
\end{gathered}
$$

We divide the proof into the next two cases.

Case 1. $c=1$.

By Eq. (4.1), it is easy to see that $\mu_{n}\left(S^{C}(n, c)\right)=0, \mu_{n-1}\left(S^{C}(n, c)\right)>n-5$ and $\mu_{2}\left(S^{C}(n, c)\right)=n-3$.

Case 2. $c \geq 2$.

Since $n-c-2>0$, by Eq. (4.1) we can conclude that $\mu_{n}\left(S^{C}(n, c)\right)=0$, $\mu_{n-1}\left(S^{C}(n, c)\right)=n-5$ and $\mu_{2}\left(S^{C}(n, c)\right)=n-3$.

Lemma 4.4. For $c \geq 1$ and $n \geq 8$, if there exists some graph $G=G^{*} \cup K_{1}$ such that $G^{*}$ is connected and $S Q(G)=S Q\left(S^{C}(n, c)\right)$, then $d_{n-1}\left(G^{*}\right)=n-3$.

Proof. By Lemmas 4.2 and 4.3, we can conclude that $n-5 \leq \mu_{n-1}\left(G^{*}\right) \leq$ $d_{n-1}\left(G^{*}\right) \leq n-2$. If $d_{n-1}\left(G^{*}\right)=n-2$, then $G^{*} \cong K_{n-1}$, and hence $S Q\left(G^{*}\right)=$ $(2 n-4, n-3, \ldots, n-3) \neq S Q\left(S^{C}(n, c)\right)$, a contradiction. We divide the proof into the next two cases.

Case 1. $d_{n-1}\left(G^{*}\right)=n-5$.

Let $H_{1}$ be the graph obtained from $K_{n-1}$ by deleting three edges, which are adjacent to the same vertex, from $K_{n-1}$. Clearly, $d_{n-1}\left(H_{1}\right)=n-5$ and $G^{*}$ is a 
subgraph of $H_{1}$. By a straightforward computation, we have

$$
\Phi\left(H_{1}, x\right)=(x-n+4)^{2}(x-n+3)^{n-5} \varphi_{4}(x),
$$

where $\varphi_{4}(x)=x^{2}-(3 n-11) x+2(n-4)(n-5)$.

It is easy to see that the roots of $\varphi_{4}(x)=0$ are

$$
\frac{3 n-11 \pm \sqrt{n^{2}+6 n-39}}{2} .
$$

By Lemma 4.1, it follows that

$$
\mu_{n-1}\left(G^{*}\right) \leq \mu_{n-1}\left(H_{1}\right)=\frac{3 n-11-\sqrt{n^{2}+6 n-39}}{2}<n-5 .
$$

On the other hand, $\mu_{n-1}\left(G^{*}\right)=\mu_{n-1}(G)=\mu_{n-1}\left(S^{C}(n, c) \geq n-5\right.$, a contradiction.

Case 2. $d_{n-1}\left(G^{*}\right)=n-4$.

Let $H_{2}$ be the graph obtained from $K_{n-1}$ by deleting two edges, which are adjacent to the same vertex, from $K_{n-1}$. Clearly, $d_{n-1}\left(H_{2}\right)=n-4$ and $G^{*}$ is a subgraph of $H_{2}$. By a straightforward computation, we have

$$
\Phi\left(H_{2}, x\right)=(x-n+4)(x-n+3)^{n-4} \varphi_{5}(x),
$$

where $\varphi_{5}(x)=x^{2}-(3 n-10) x+2(n-4)^{2}$.

It is easy to see that the roots of $\varphi_{5}(x)=0$ are

$$
\frac{3 n-10 \pm \sqrt{n^{2}+4 n-28}}{2} .
$$

By Lemma 4.1, it follows that

$$
\mu_{n-1}\left(G^{*}\right) \leq \mu_{n-1}\left(H_{2}\right)=\frac{3 n-10-\sqrt{n^{2}+4 n-28}}{2}<n-5 .
$$

On the other hand, $\mu_{n-1}\left(G^{*}\right)=\mu_{n-1}(G)=\mu_{n-1}\left(S^{C}(n, c) \geq n-5\right.$, a contradiction.

By combining the above arguments, we can conclude that $d_{n-1}\left(G^{*}\right)=n-3$.

LEMMA 4.5. If $c=0$ and $n \neq 4$, then $S^{C}(n, c)$ is determined by its signless Laplacian spectrum

Proof. If $1 \leq n \leq 3$, it is easily checked the result follows. Thus, we may assume that $n \geq 5$ in the following. Suppose that there exists some graph $G$ such that $S Q(G)=S Q\left(S^{C}(n, c)\right)$. Note that $S^{C}(n, c)=K_{n-1} \cup K_{1}$. Then, $\mu_{n}(G)=$ $\mu_{n}\left(K_{n-1} \cup K_{1}\right)=0$ and $\mu_{1}(G)=\mu_{1}\left(K_{n-1} \cup K_{1}\right)=2(n-2)$. 
If $G$ is connected, since $\mu_{n}(G)=0$, by Lemma 3.13 it follows that $G$ is bipartite. Lemma 2.2 implies that $\mu_{1}(G)=\lambda_{1}(G) \leq n<2(n-2)$, a contradiction. Thus, $G$ is disconnected and hence $d_{1}(G) \leq n-2$. Since $\mu_{1}(G)=2(n-2)$, by Lemma 3.7 we can conclude that $G \cong K_{n-1} \cup K_{1}=S^{C}(n, c)$.

REMARK 4.6. It is easily checked that $S Q\left(K_{3} \cup K_{1}\right)=S Q\left(K_{1,3}\right)$. Thus, $S^{C}(n, c)$ is not determined by its signless Laplacian spectrum when $c=0$ and $n=4$.

THEOREM 4.7. If $c \geq 0$, then $S^{C}(n, c)$ is determined by its signless Laplacian spectrum except for the case of $c=0$ and $n=4$.

Proof. If $c=0$, by Lemma 4.5 and Remark 4.6, the result follows. If $c \geq 1$ and $3 \leq n \leq 7$, it is easily checked the result follows by the aid of computer. Thus, we may assume that $n \geq 8$ and $c \geq 1$ in the sequel. Now suppose there exists some graph $G$ such that $S Q(G)=S Q\left(S^{C}(n, c)\right)$. We only need to prove the following facts:

Fact 1. $G=G^{*} \cup K_{1}$, where $G^{*}$ is connected.

Proof of Fact 1. We first claim that $G$ is disconnected. Suppose to the contrary, $G$ is connected. By Lemma 4.3, we have $\mu_{n}(G)=\mu_{n}\left(S^{C}(n, c)\right)=0$. Thus, $G$ is bipartite by Lemma 3.13. So, $\mu_{1}(G) \leq n$ follows from Lemma 2.2. But $\mu_{1}(G)=$ $\mu_{1}\left(S^{C}(n, c) \geq 2(n-3)>n\right.$ by Lemma 4.3 , a contradiction. Thus, $G$ is disconnected.

Let $G_{1}$ be the greatest connected component, i.e., the connected component with largest number of vertices, of $G$. Since $\mu_{n}(G)=0$ and $\mu_{n-1}(G)=\mu_{n-1}\left(S^{C}(n, c)\right) \geq$ $n-5>0$, by Lemmas 3.13 and 4.2 we can conclude that $G$ has exactly one bipartite component and $\left|V\left(G_{1}\right)\right| \geq n-4$. Moreover, Lemma 4.3 implies that $\mu_{1}(G)=$ $\mu_{1}\left(S^{C}(n, c) \geq 2(n-3)\right.$, thus $\left|V\left(G_{1}\right)\right| \geq n-2$ by Lemma 3.7.

If $\left|V\left(G_{1}\right)\right|=n-2$, since $G$ has exactly one bipartite component, we can deduce that $G=G_{1} \cup K_{2}$. Then $G$ has 2 as its signless Laplacian eigenvalue. On the other hand, Lemma 4.3 implies that $\mu_{n-1}(G)=\mu_{n-1}\left(S^{C}(n, c) \geq n-5>2\right.$, a contradiction. Thus, $\left|V\left(G_{1}\right)\right|=n-1$ and hence Fact 1 follows.

Fact 2. $G \cong S^{C}(n, c)$.

Proof of Fact 2. By Fact 1 and Lemma 4.4, it follows that $G=G^{*} \cup K_{1}$, where $G^{*}$ is connected with $d_{n-1}\left(G^{*}\right)=n-3$. By Lemma 3.11, it follows that $G^{*}$ has $n-2 c-1$ vertices of degree $n-2$ and $2 c$ vertices of degree $n-3$, then $G \cong S^{C}(n, c)$ follows.

This completes the proof of this result.

Acknowledgment. The authors are very grateful to the anonymous referee for his valuable comments and suggestions, which led to an improvement of the original manuscript. 


\section{REFERENCES}

[1] B. Borovićanin and M. Petrović. On the index of cactuses with $n$ vertices. Publ. Inst. Math. (Beograd), 79(93):13-18, 2006.

[2] A.E. Brouwer and W.H. Haemers. A lower bound for the Laplacian eigenvalues of a graph-Proof of a conjecture by Guo. Linear Algebra Appl., 429(8):2131-2135, 2008.

[3] D.M. Cardoso, D. Cvetković, P. Rowlinson, and S.K. Simić. A sharp lower bound for the least eigenvalue of the signless Laplacian of a non-bipartite graph. Linear Algebra Appl., 429:2770-2780, 2008.

[4] D.M. Cvetković, M. Doob, and H. Sachs. Spectra of Graphs. Theory and Application, second edition. VEB Deutscher Verlag der Wissenschaften, Berlin, 1982.

[5] D. Cvetković, P. Rowlinson, and S. K. Simić. Signless Laplacians of finite graphs. Linear Algebra Appl., 423:155-171, 2007.

[6] E.R. van Dam and W.H. Haemers. Which graphs are determined by their spectrum? Linear Algebra Appl., 373:241-272, 2003.

[7] K.C. Das. The Laplacian spectrum of a graph. Comput. Math. Appl., 48:715-724, 2004.

[8] K.C. Das. On conjectures involving second largest signless Laplacian eigenvalue of graphs. Linear Algebra Appl., 432:3018-3029, 2010.

[9] M. Doob and W.H. Haemers. The complement of the path is determined by its spectrum. Linear Algebra Appl., 356:57-65, 2002.

[10] Y.Z. Fan, B.S. Tam, and J. Zhou. Maximizing spectral radius of unoriented Laplacian matrix over bicyclic graphs of a given order. Linear Multilinear Algebra, 56(4):381-397, 2008.

[11] W.H. Haemers. Interlacing eigenvalues and graphs. Linear Algebra Appl., 226/228:593-616, 1995.

[12] S. Kirkland. Completion of Laplacian integral graphs via edge addition. Discrete Math., 295:7590, 2005.

[13] B.L. Liu, Z.B. Chen, and M.H. Liu. On graphs with the largest Laplacian index. Czechoslovak Math. J., 58:949-960, 2008.

[14] H.Q. Liu and M. Lu. A unified approach to extremal cacti for different indices. MATCH Commun. Math. Comput. Chem., 58:183-194, 2007.

[15] M.H. Liu and B.L. Liu. Some results on the Laplacian spectrum. Comput. Math. Appl., 59:3612-3616, 2010.

[16] M.H. Liu and B.L. Liu. The signless Laplacian spread. Linear Algebra Appl., 432(2-3):505-514, 2010.

[17] R. Merris. Laplacian matrices of graphs: a survey. Linear Algebra Appl., 197/198:143-176, 1994.

[18] R. Merris. Laplacian graph eigenvectors. Linear Algebra Appl., 278:221-236, 1998.

[19] Y.L. Pan. Sharp upper bounds for the Laplacian graph eigenvalues. Linear Algebra Appl., 355:287-295, 2002.

[20] Z. Radosavljević and M. Rašajski. A class of reflexive cactuses with four cycles. Univ. Beograd. Publ. Elektrotehn. Fak., Ser. Mat., 14:64-85, 2003.

[21] X.L. Shen and Y.P. Zhang. The star and all starlike trees with largest degree 3 are determined by their spectra. J. Nat. Sci. Hunan Norm. Univ., 28:17-20, 2005.

[22] B.S. Tam, Y.Z. Fan, and J. Zhou. Unoriented Laplacian maximizing graphs are degree maximal. Linear Algebra Appl., 429:735-758, 2008.

[23] X.L. Zhang and H.P. Zhang. Some graphs determined by their spectra. Linear Algebra Appl., 431:1443-1454, 2009.

[24] Y.P. Zhang, X.G. Liu, B.Y. Zhang, and X.R. Yong. The lollipop graph is determined by its Q-spectrum. Discrete Math., 309:3364-3369, 2009. 